\title{
Modeling and Vibration Analysis of Spinning Hard Disk and Head Assembly *
}

\author{
Kao-An LIN ${ }^{* *}$ and Shyh-Chin HUANG ${ }^{* *}$ \\ **Department of Mechanical Engineering, National Taiwan University of Science and Technology, 43, \\ Kee-Lung Rd, Sec.4, Taipei, Taiwan 106 \\ E-mail: schuang@mail.ntust.edu.tw
}

\begin{abstract}
A read/write head assembly attached to a spinning disk was modeled and investigated through a different approach, in which the head assembly was represented by a suspension arm with an attached mass and an air spring (film) at its free end. The receptance method was applied to connect the spinning disk and the head assembly. The natural frequencies and mode shapes of the combined spinning disk-fixed head assembly as a whole were then interpreted. Numerical results showed that the head assembly induced extra modes from a single disk. Even for just weak coupling between disk and head, the bifurcations of mode shapes were very obvious, but the changes of natural frequencies were slight. The effects on frequency changes due to head's flexibility, air spring constant, head's location, and spinning speed were examined as well. Disk's spinning speed was found to pull the disk-head frequency loci to pass through the crossings of single disk's frequency loci and induce curve veering phenomenon.
\end{abstract}

Key words: Spinning Disk Vibration, Disk-Head Assembly, Receptance Method

\section{Introduction}

Spinning disks have been widespread in engineering applications, e.g., saw blades, turbine rotors, and computer disks. Numerous researches regarding the vibrations of spinning disk have been published, e.g., Lamb and Southwell ${ }^{(1)}$ derived the equations for a spinning disk and solved for its natural frequencies and mode shapes. Mote ${ }^{(2)}$ used the Rayleigh-Ritz method to solve for a disk with initial stresses. Adams ${ }^{(3)}$ discussed the critical speeds of a spinning disk. Eversman and Cloyd ${ }^{(4)}$ investigated the effect of hub coupling on the transverse free vibration of a spinning disk. Phylactopoulos and Adams ${ }^{(5,6)}$ analyzed the transverse free and forced vibration of a rectangular orthotropic spinning disk. Chung et al. ${ }^{(7)}$ utilized the Galerkin method to discuss the effects of axis misalignment on the natural frequencies for spinning disk. Lee and $\mathrm{Ng}^{(8)}$ used the assumed mode method and the Lagrangian approach to solve for natural frequencies and critical speeds of a spinning disk of linearly and exponentially varying thickness. Eid and Adams ${ }^{(9)}$ estimated the critical speeds and the response of a spinning disk subject to a stationary load using the Mindlin plate theory.

Sinha dealt with the free vibration of a spinning annular disk with uniformly distributed masses $^{(10)}$ and a single ring ${ }^{(11)}$ attached to disk's outer edge by the Rayleigh-Ritz technique. DasGupta and Hagedorn ${ }^{(12)}$ studied critical speeds of a spinning thin axisymmetric annular disk with an external ring. Yang ${ }^{(13)}$ analyzed the vibration of a spinning annular disk with coupled translational and rotational rigid body motion. Young and $\operatorname{Lin}^{(14)}$ studied the free vibration and elastic stability of a spinning annular disk transversely in contact with a stationary oscillating unit which consisted of two parallel combinations of springs and dampers. Some studies focused on the influence of disk's flexibility to whole system, e.g., Chivens and Nelson ${ }^{(15)}$ investigated the influence of disk flexibility on bending natural frequencies and critical speeds of a flexible shaft-disk system using the Laplace transform method. Shahab and Thomas ${ }^{(16)}$ discussed the coupling effects of shaft and disk flexibility 
on a shaft-multiple disks system using FEM. Yang and Huang ${ }^{(17)}$ used the assumed mode method to analyze the effects of disk flexibility on the coupling vibration of a rotating shaft-disk-blade system.

Since early 70's, due to the advance of computer technology, significant researches on spinning disks had been applied for simulating computer hard disk drives. Most of the researchers treated the head assembly as an external load to the disk and solved for the disk's response. Among them, Benson and Bogy ${ }^{(18)}$ treated the head as a stationary transverse load. Stahl and $\operatorname{Iwan}^{(19,20)}$ looked the disk as a rigid element but of two/three rotational degree-of-freedom in which the head was modeled as a counter-spinning mass. Iwan and Moeller $^{(21)}$ investigated the free vibration and stability of a spinning disk with a stationary mass-spring-damper load system. Shen and Mote ${ }^{(22)}$ modeled the head as a spring-mass-dashpot (SMD). Chen and Bogy examined the natural frequencies and stability of a spinning disk with a stationary load system ${ }^{(23)}$, and then extended it to allow disk's rigid body tilting ${ }^{(24)}$. Liang et al. ${ }^{(25)}$ studied the vibration and stability of a spinning polar orthotropic disk with a concentrated transverse load system. Huang and $\mathrm{Chiou}^{(26)}$ treated the head as a moving force and discussed its traveling effects and resonant phenomena. Lately Yu and Huang ${ }^{(27)}$ explored the damping effects of a CLD on spring-mass-plate system.

Instead of treating the head assembly as an external load, the present research takes the spinning disk and head assembly as a whole and develops an efficient approach to its vibration analysis. The head assembly is not modeled as simple as a SMD as previously. Instead, it is modeled as a clamped beam with a tip mass. Between the tip mass and spinning disk there interacts an air spring. This model closely resembles the real head assembly system. The most crucial part encountered in the spinning disk-head assembly is the modeling of the interactions between spinning disk and stationary read/write head. The difficulty arises from this approach is the connection between stationary (head assembly) and spinning (disk) elements. The authors employ the structure combination technique and eventually, a very neat expression for the natural frequencies of spinning disk-head assembly is obtained. The mode shapes and natural frequencies vary with the air spring constant, head location, head's flexibility, and spinning speed are then looked into numerically.

\section{Frequency equation and mode shapes}

Figure 1 shows a schematic diagram of a spinning hard disk with read/write head assembly. The read/write head assembly is modeled as a cantilevered beam with an attached mass-air spring as Fig. 2 shows. Utilizing the receptance method, this system is divided into two main subbodies: (A) the spinning disk and (B) the read/write head assembly, as shown in Fig. 1(b). Note that the contact point is varying due to disk spinning but looks stationary from an inertia observer, provided the disk is homogenous and uniform.

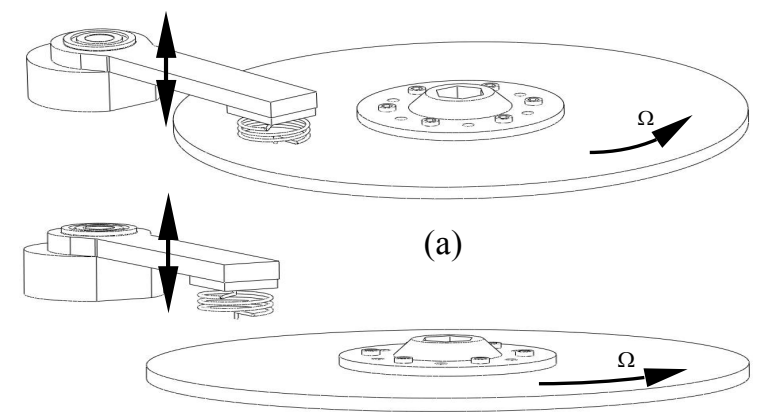

(b)

Fig. 1: Schematic diagram of a spinning disk and head assembly. 


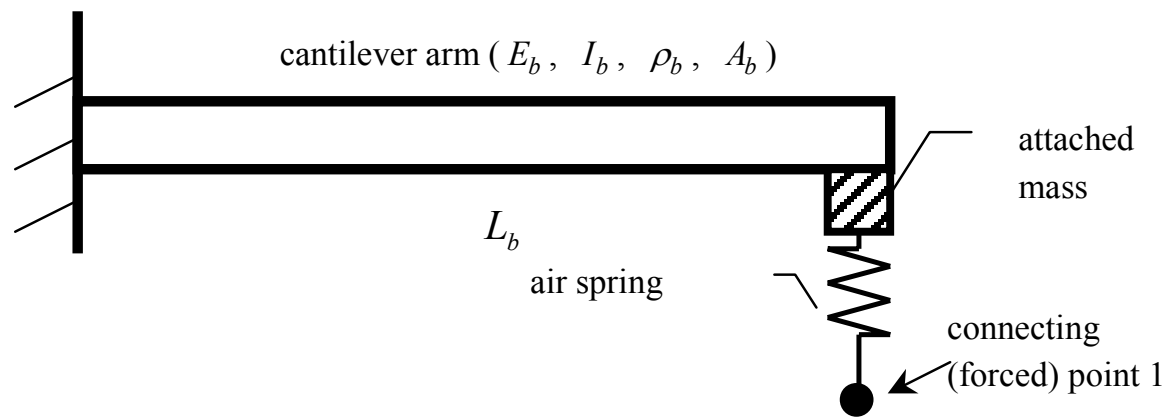

Fig. 2: Schematic diagram of a head assembly.

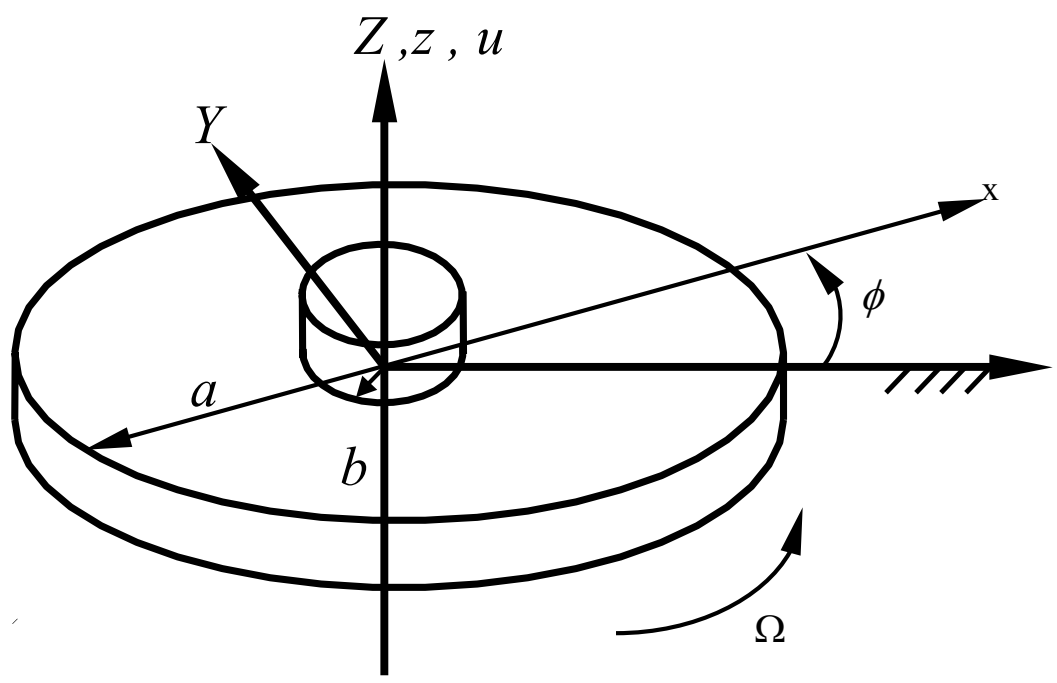

Fig. 3: Schematic diagram and coordinates of a spinning disk.

Figure 3 denotes a spinning disk, in which (X,Y,Z), an inertia frame; $a$, the outer radius; $b$, the inner radius; $\Omega$, the spinning speed; and $\phi$, the angular coordinate with respect to the inertia frame. The equation of motion of the spinning disk in terms of its transverse displacement $u(r, \phi, t)$ with respect to a stationary observer is then derived ${ }^{(28)}$

$$
\begin{aligned}
D\left(\frac{\partial^{2}}{\partial r^{2}}+\frac{\partial}{r \partial r}\right. & \left.+\frac{1}{r^{2}} \frac{\partial^{2}}{\partial \phi^{2}}\right)^{2} u-\frac{h}{r}\left[\frac{\partial}{\partial r}\left(r \sigma_{r} \frac{\partial u}{\partial r}\right)+\frac{\partial}{r \partial \phi}\left(\sigma_{\phi} \frac{\partial u}{\partial \phi}\right)\right] \\
& +\rho h\left[\frac{\partial^{2} u}{\partial t^{2}}+2 \Omega \frac{\partial^{2} u}{\partial \phi \partial t}+\Omega^{2} \frac{\partial^{2} u}{\partial \phi^{2}}\right]=p(r, \phi, t)
\end{aligned}
$$

where $D=E h^{3} / 12\left(1-v^{2}\right)$, the bending rigidity; $\rho$, density; $h$, thickness; $v$, Poisson's ratio; $p$ pressure type loading; $\sigma_{r}$ and $\sigma_{\phi}$, initial stresses due to spin,

$$
\begin{aligned}
& \sigma_{r}(r)=\frac{(3+v)}{8} \rho \Omega^{2}\left(a^{2}-r^{2}\right)+\frac{\rho \Omega^{2} b^{2}(1-v)\left[a^{2}(3+v)-b^{2}(1+v)\right]}{8\left[b^{2}(1-v)+a^{2}(1+v)\right]}\left(\frac{a^{2}}{r^{2}}-1\right) \\
& \sigma_{\phi}(r)=\frac{\rho \Omega^{2}}{8}\left[(3+v) a^{2}-(1+3 v) r^{2}\right]-\frac{\rho \Omega^{2} b^{2}(1-v)\left[a^{2}(3+v)-b^{2}(1+v)\right]}{8\left[b^{2}(1-v)+a^{2}(1+v)\right]}\left(\frac{a^{2}}{r^{2}}+1\right)
\end{aligned}
$$

The disk is assumed clamped inside and free outside, i.e.,

$$
\left\{\begin{array}{l}
M_{r r}(a, \phi, t)=0 \\
V_{r r}(a, \phi, t)=0 \\
u(b, \phi, t)=0 \\
\frac{\partial u(b, \phi, t)}{\partial r}=0
\end{array}\right.
$$

where the bending moments and effective shear forces are 
$M_{r r}=-D\left[\frac{\partial^{2} u}{\partial r^{2}}+v\left(\frac{1}{r} \frac{\partial u}{\partial r}+\frac{1}{r^{2}} \frac{\partial^{2} u}{\partial \phi^{2}}\right)\right]$

$V_{r r}=Q_{r}+\frac{1}{r} \frac{\partial M_{r \phi}}{\partial \phi}$

$Q_{r}=-D \frac{\partial}{\partial r}\left(\nabla^{2} u\right)$

$M_{r \theta}=-D(1-v) \frac{\partial}{\partial r}\left(\frac{1}{r} \frac{\partial u}{\partial \phi}\right)$

By the traveling modes expansion ${ }^{(28)}$, the displacement function is assumed to be

$$
\begin{aligned}
u(r, \phi, t) & =\sum_{m=0}^{M} \sum_{n=0}^{N} \eta_{m n}(t) \cdot R_{m n}(r) e^{i\left(n \phi+\omega_{m n} t\right)} \\
& =\sum_{m=0}^{M} \sum_{n=0}^{N}\left[q_{m n}^{c}(t) \cos n \phi+q_{m n}^{s}(t) \sin n \phi\right] R_{m n}(r),
\end{aligned}
$$

where $m$, nodal circle number; $n$, nodal diameter number; $M, N$, the numbers of terms deemed necessary for satisfactory accuracy; $R_{m n}(r)$ is disk's radial function and is here chosen the beam function, i.e.,

$$
\begin{aligned}
R_{m n}(r)= & {\left[\sin \beta_{m n}(r-b)-\sinh \beta_{m n}(r-b)\right] } \\
& +\alpha_{m n}\left[\cos \beta_{m n}(r-b)-\cosh \beta_{m n}(r-b)\right]
\end{aligned}
$$

Note that Eq. (10) by itself satisfies the inner clamped boundary but $\alpha_{m n}, \beta_{m n}$ are two parameters chosen to satisfy the outer boundary.

Substitute Eq. (9) into Eq. (1), employ the Galerkin's method, and utilize the orthogonality of trigonometric functions, the participation factors for each $n$ are decoupled and solved for from the following equations,

$\left[\mathrm{T}_{s m 0}\right]\left[\ddot{q}_{m 0}^{c}\right\}+\left[\Phi_{s m 0}\right]\left\{q_{m 0}^{c}\right\}=\left\{Q_{s 0}^{c}\right\}$ for $n=0$

$\left.\left.\left[\mathrm{T}_{s m n}\right]\right] \ddot{q}_{m n}^{c}\right\}+2 n \Omega\left[\mathrm{T}_{s m n}\right]\left\{\dot{q}_{m n}^{s}\right\}+\left(\left[\Phi_{s m n}\right]-n^{2} \Omega^{2}\left[\mathrm{~T}_{s m n}\right]\right)\left\{q_{m n}^{c}\right\}=\left\{Q_{s n}^{c}\right\}$

$\left.\left.\left.\left.\left[\mathrm{T}_{s m n}\right]\right\} \ddot{q}_{m n}^{s}\right\}-2 n \Omega\left[\mathrm{T}_{s m n}\right]\right\} \dot{q}_{m n}^{c}\right\}+\left(\left[\Phi_{s m n}\right]-n^{2} \Omega^{2}\left[\mathrm{~T}_{s m n}\right]\right)\left\{\begin{array}{c}s \\ q_{m n}\end{array}\right\}=\left\{Q_{s n}^{s}\right\}$

where $s, m=0,1, \ldots, M$. The above matrices are of dimension $(M+1) \times(M+1)$ and the entries are of the forms:

$$
\begin{aligned}
\mathrm{T}_{s m n}= & \int_{b}^{a} R_{m n}(r) R_{s n}(r) r d r \\
\Phi_{s m n}= & \frac{1}{\rho h}\left\{D \int_{b}^{a}\left\{\left[\left(\frac{d^{2}}{d r^{2}}+\frac{d}{r d r}-\frac{n^{2}}{r^{2}}\right)^{2} R_{m n}(r)\right] \cdot R_{s n}(r)\right\} r d r\right. \\
& \left.-h \int_{b}^{a}\left\{\left[\frac{d}{d r}\left(r \sigma_{r} \frac{d R_{m n}(r)}{d r}\right)-\frac{n^{2}}{r} \sigma_{\phi} R_{m n}(r)\right] \cdot R_{s n}(r)\right\} d r\right\} \\
Q_{s 0}^{c}= & \frac{1}{2 \rho h \pi} \int_{b}^{a} \int_{0}^{2 \pi} R_{s 0}(r) p(r, \phi, t) r d \phi d r \\
Q_{s n}^{c}= & \frac{1}{\rho h \pi} \int_{b}^{a} \int_{0}^{2 \pi} R_{s n}(r) p(r, \phi, t) \cos (n \phi) r d \phi d r \\
Q_{s n}^{s}= & \frac{1}{\rho h \pi} \int_{b}^{a} \int_{0}^{2 \pi} R_{s n}(r) p(r, \phi, t) \sin (n \phi) r d \phi d r
\end{aligned}
$$

Equations (11-13) enable us to solve for, individually, the participation factors of each $n$ number. The selection of $M$ relies on the frequency range of interest. For cases of lower frequency range take only the zero $(s=m=0)$ nodal circle modes may be enough and Eqs. (11-13) are subsequently simplified to be

$$
\ddot{q}_{0}^{c}(t)+\Psi_{0}^{2} q_{0}^{c}(t)=\frac{Q_{0}^{c}}{\mathrm{~T}_{0}}, \quad n=0
$$


$\left\{\begin{array}{l}\ddot{q}_{n}^{c} \\ \ddot{q}_{n}^{s}\end{array}\right\}+\left[\begin{array}{cc}0 & 2 n \Omega \\ -2 n \Omega & 0\end{array}\right]\left\{\begin{array}{l}\dot{q}_{n}^{c} \\ \dot{q}_{n}^{s}\end{array}\right\}+\left[\begin{array}{cc}\Psi_{n}^{2}-n^{2} \Omega^{2} & 0 \\ 0 & \Psi_{n}^{2}-n^{2} \Omega^{2}\end{array}\right]\left\{\begin{array}{l}q_{n}^{c} \\ q_{n}^{s}\end{array}\right\}=\frac{1}{\mathrm{~T}_{n}}\left\{\begin{array}{l}Q_{n}^{c} \\ Q_{n}^{s}\end{array}\right\}, n \neq 0$

where $\Psi_{n}^{2}=\Phi_{n} / \mathrm{T}_{n}$.

Solve the above equations for natural frequencies, one obtains $\omega_{n 1}=n \Omega-\Psi_{n}$

$\omega_{n 2}=n \Omega+\Psi_{n}$

where $\omega_{n 1,2}$ are the natural frequencies of the $(0, n)$ modes, one forward and one backward, as viewed by a stationary observer. It can be proven that $\Psi_{n}$ is exactly the disk-based (rotating) natural frequency.

The receptance method is now applied for joining the spinning disk and head assembly. Receptance method requires the calculations of harmonically forced response. It is hence assumed that a unit harmonic point force acts at $\left(r_{0}, \phi_{0}\right)$, i.e.,

$p(r, \phi, t)=\frac{1}{r} \delta\left(\phi-\phi_{0}\right) \delta\left(r-r_{0}\right) e^{i \omega t}$

Subsequently, the disk's response is solved to be

$$
\begin{array}{r}
u(r, \phi, t)=\sum_{n=0}^{N} \frac{R_{n}\left(r_{0}\right) R_{n}(r) e^{i \omega t}}{\mu \rho h \mathrm{~T}_{n}\left(\omega^{2}-\omega_{n 1}^{2}\right)\left(\omega_{n 2}^{2}-\omega^{2}\right)}\left\{\left(\omega_{n 1} \omega_{n 2}+\omega^{2}\right) \cos n\left(\phi-\phi_{0}\right)\right. \\
\left.\left.-i \mid \omega\left(\omega_{n 2}+\omega_{n 1}\right) \sin n\left(\phi-\phi_{0}\right)\right]\right\}, \quad \mu=\left\{\begin{array}{cc}
2 \pi & n=0 \\
\pi & n \neq 0
\end{array}\right.
\end{array}
$$

The receptance $\alpha_{i j}$ is the ratio of displacement response at $\left(r_{i}, \phi_{i}\right)$ to the harmonic point force at $\left(r_{j}, \phi_{j}\right)$ and it is calculated to be

$\alpha_{i j}=\mathfrak{R}_{i j}+i \Im_{i j}$,

with

$\Re_{i j}=\sum_{n=0}^{N} \frac{R_{n}\left(r_{i}\right) R_{n}\left(r_{j}\right)\left\{\left(\omega_{n 1} \omega_{n 2}+\omega^{2}\right) \cos \left[n\left(\phi_{i}-\phi_{j}\right)\right]\right\}}{\mu \rho h \mathrm{~T}_{n}\left(\omega^{2}-\omega_{n 1}^{2}\right)\left(\omega_{n 2}^{2}-\omega^{2}\right)}$,

$\mathfrak{J}_{i j}=\sum_{n=0}^{N} \frac{-R_{n}\left(r_{i}\right) R_{n}\left(r_{j}\right)\left\{\omega\left(\omega_{n 2}+\omega_{n 1}\right) \sin \left[n\left(\phi_{i}-\phi_{j}\right)\right]\right\}}{\mu \rho h \mathrm{~T}_{n}\left(\omega^{2}-\omega_{n 1}^{2}\right)\left(\omega_{n 2}^{2}-\omega^{2}\right)}$.

Note that the cross receptances for a spinning structure are in complex conjugate pairs, i.e., $\alpha_{i j}=\tilde{\alpha}_{j i}$. The direct receptance yet is always a real number, i.e.,

$\alpha_{i i}=\sum_{n=0}^{N} \frac{R_{n}^{2}\left(r_{i}\right)\left(\omega_{n 1} \omega_{n 2}+\omega^{2}\right)}{\mu \rho \mathrm{T}_{n}\left(\omega^{2}-\omega_{n 1}^{2}\right)\left(\omega_{n 2}^{2}-\omega^{2}\right)}$

In a similar manner, the receptance $\beta_{i j}^{b}$ of a cantilever arm at its tip end is solved to be

$$
\beta_{i j}^{b}=\sum_{n=1}^{\infty} \frac{\phi_{n}\left(x_{i}\right) \phi_{n}\left(x_{j}\right)}{\rho_{b} A_{b}\left(\omega_{n}^{b^{2}}-\omega^{2}\right)\left(\int_{0}^{L_{b}} \phi_{n}^{2}(x) d x\right)}
$$

where $\phi_{n}(x)$ is the $n^{\text {th }}$ mode of a clamped-free beam. $L_{b}, \rho_{b}, A_{b}$ and $\omega_{n}^{b}$ denote the beam's length, density, cross-sectional area and $n^{\text {th }}$ eigen-frequency, respectively. Subsequently, the receptances of the head assembly (beam-mass-air spring), as shown in Fig. 2 , at its tip (denoted \#1) is

$\beta_{11}=\frac{\beta_{11}^{b} \beta^{m}}{\beta_{11}^{b}+\beta^{m}}+\beta^{s}$.

Where

$$
\beta_{11}^{b}=\sum_{n=1}^{\infty} \frac{\phi_{n}^{2}\left(x_{1}\right)}{\rho_{b} A_{b}\left(\omega_{n}^{b^{2}}-\omega^{2}\right)\left(\int_{0}^{L_{b}} \phi_{n}^{2}(x) d x\right)}
$$




$$
\left\{\begin{array}{c}
\beta^{m}=\frac{-1}{M \omega^{2}} \\
\beta^{s}=\frac{1}{k}
\end{array}\right.
$$

$M$ and $k$ are the head mass and air spring constant, respectively.

The frequency equation for the spinning disk-head assembly system as Fig. 1 shows is hence derived to be

$\alpha_{11}(\omega)+\beta_{11}(\omega)=\sum_{n=0}^{N} \frac{R_{n}^{2}\left(r_{1}\right)\left(\omega_{n 1} \omega_{n 2}+\omega^{2}\right)}{\mu h \mathrm{~T}_{n}\left(\omega^{2}-\omega_{n 1}^{2}\right)\left(\omega_{n 2}^{2}-\omega^{2}\right)}+\frac{\beta_{11}^{b} \beta^{m}}{\beta_{11}^{b}+\beta^{m}}+\beta^{s}=0$.

Equation (33) provides us to solve for the natural frequencies, said $\omega_{k}$ 's, of the spinning disk-head assembly and the associated mode shape can be calculated from the following equation,

$\gamma\left(x_{i}\right)=\left.\frac{\beta_{i 1}^{b} \beta^{m}}{\beta_{11}^{b}+\beta^{m}}\right|_{\omega=\omega_{k}}$

$\gamma\left(x_{i}\right)$ represents the deflective shapes of the head assembly.

The disks' mode shapes are derived from Eq. (24) to be

$$
\begin{gathered}
u\left(r_{i}, \phi_{i}, t\right)=\sum_{n=0}^{N} \frac{R_{n}\left(r_{i}\right) R_{n}\left(r_{1}\right) e^{i \omega_{k} t}}{\mu \rho \mathrm{T}_{n}\left(\omega_{k}^{2}-\omega_{n 1}^{2}\right)\left(\omega_{n 2}^{2}-\omega_{k}^{2}\right)}\left\{\left(\omega_{n 1} \omega_{n 2}+\omega_{k}^{2}\right) \cos n\left(\phi_{i}-\phi_{1}\right)\right. \\
\left.-i\left[\omega_{k}\left(\omega_{n 2}+\omega_{n 1}\right) \sin n\left(\phi_{i}-\phi_{1}\right)\right]\right\}
\end{gathered}
$$

Note that the modes described by Eq. (35) are the so-called traveling modes due to disk's spinning and they will become stationary if $\Omega=0$.

\section{Numerical Results}

In the following, a typical hard disk-head system is illustrated. The geometrical and material properties of the disk and head assembly are given in Table 1 . Table 1 also shows the obtained first four natural frequencies $(m=0, n=1,0,2,3)$ of the disk at $\Omega=0$. In order to present the results in dimensionless forms, the following parameters are defined: $\omega^{*}=\omega\left(\rho h a^{4} / D\right)^{1 / 2}, \Omega^{*}=\Omega\left(\rho h a^{4} / D\right)^{1 / 2}, k^{*}=k /\left[D /(a-b)^{2}\right]$, respectively represent the normalized natural frequency, spinning speed, and air spring constant.

Table 1: Disk and head assembly's properties and natural frequencies.

\begin{tabular}{ccc}
\hline & Disk & Cantilever arm \\
\hline Density & $\rho=2.66 \times 10^{3} \mathrm{~kg} / \mathrm{m}^{3}$ & $\rho_{b}=7.80 \times 10^{3} \mathrm{~kg} / \mathrm{m}^{3}$ \\
\hline Modulus & $E=70.6 \times 10^{9} \mathrm{~N} / \mathrm{m}^{2}$ & $E_{b}=20.69 \times 10^{10} \mathrm{~N} / \mathrm{m}^{2}$ \\
\hline Poisson ratio & $v=0.3$ & - \\
\hline Thickness & $h=0.38 \mathrm{~mm}$ & $h_{b}=1.1 \mathrm{~mm}$ \\
\hline Outer radius & $a=47.50 \mathrm{~mm}$ & - \\
\hline Inner radius & $b=15.50 \mathrm{~mm}$ & - \\
\hline Width & - & $W_{b}=4.5 \mathrm{~mm}$ \\
\hline Length & - & $L_{b}=25 \mathrm{~mm}$ \\
\hline Attached mass & - & $M=0.07056 \times 10^{-3} \mathrm{~kg}$ \\
\hline Natural frequencies & $7.2957,7.3050,8.6979$, & - \\
$\omega^{*}$ & 13.6846 & \\
\hline
\end{tabular}

The coupling between disk and head assembly is usually weak as far as a computer hard disk is addressed. Weak coupling is expected to alter the natural frequencies slightly. The authors intend to emphasize the coupling influence of head assembly on disk's mode 
shapes so that a moderate spring constant of $k^{*}=50$ is selected in the following examples. The effects due to spring's variation are then illustrated through separate figures.

It is of first interest to realize how the head assembly affects the disk's frequencies. Figure 4 shows the changes of natural frequencies of the disk-head assembly. It is found that the original disk's natural frequencies (in hollow symbols) split into two except the $n=0$ mode. The abscissa, not drawn in a linear scale, denotes the natural frequencies (for $n=1,0,2,3, . ., 7$ ), and the head assembly's first bending frequency is marked separately with a $\Delta$ symbol. This phenomenon is not surprising at all since every spinning disk exists two natural modes (cosine and sine) for each $n \neq 0$ number. When the head assembly is attached to the disk, one mode, said $\cos n \theta$, tends to orient itself such that the attachment passes through a node line and causes the disk's frequency unchanged. Simultaneously, the second mode, $\sin n \theta$, appears to have the attachment being an anti-node such that the head assembly's extra stiffness promotes the natural frequency. Numerical results also show that the head assembly increases the disk's natural frequencies only up to $n=4$ modes. For $n>5$ modes the disk's natural frequencies, on the contrary, decrease. This phenomenon consists with the theory of structure combination, which states that the combined frequency should fall within two substructures' frequencies.

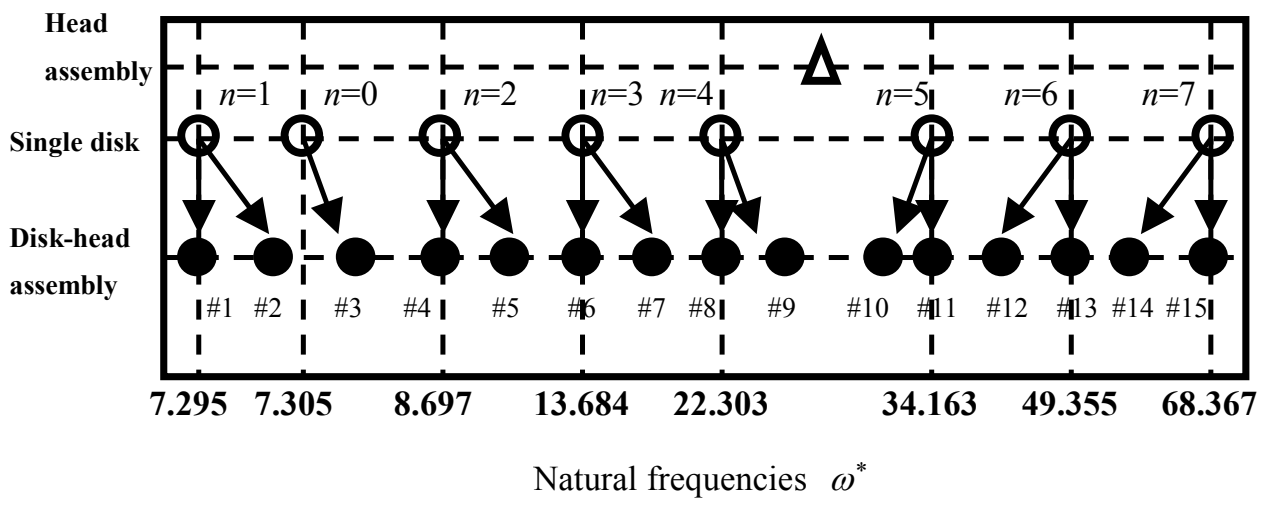

Fig. 4. Frequency bifurcations of spinning disk-head assembly.

Figure 5 shows the induced modes in an ascending order of $n=1,0,2, \ldots, 7$. The numbers inside the brackets, denote the mode number and its corresponding $\omega^{*}$. The head assembly's attachment is marked with a solid dot on disk. The left plot shows the magnified transverse deformation of disk-head assembly at the cross section of attachment, and the disk deformation contour is shown on its right. From disk's deformation, it is obvious that the head assembly twists the nodal diameters and causes mode localization, particularly significant on low $n$ modes, e.g., the $n=0$ mode (\#3) disappears and it is replaced by a mode resembling $n=1$ mode. It is also seen that the disk and head deformation can be in or out of phase. The head arm in the shown cases undergoes negligible bending at low $n$ modes but it deforms in its first mode (\#12, \#14) at higher $n$ numbers. The authors at this stage can draw a conclusion that the significant effect resulted from head assembly, even for weak coupling, is the induced disk modes rather than the changes of disk's natural frequencies.

Figure 6 shows an extreme case where the head assembly is almost rigidly (no air spring) attached to the disk. From the results, one sees that all the natural frequencies are lifted and the head attachment becomes a node of the disk. The modes shown above were for non-spinning disk. The authors have no intension to show spinning modes because it has been proven that the modes of a spinning disk were just the traveling of the non-spinning modes, one forward one backward with different speeds. 

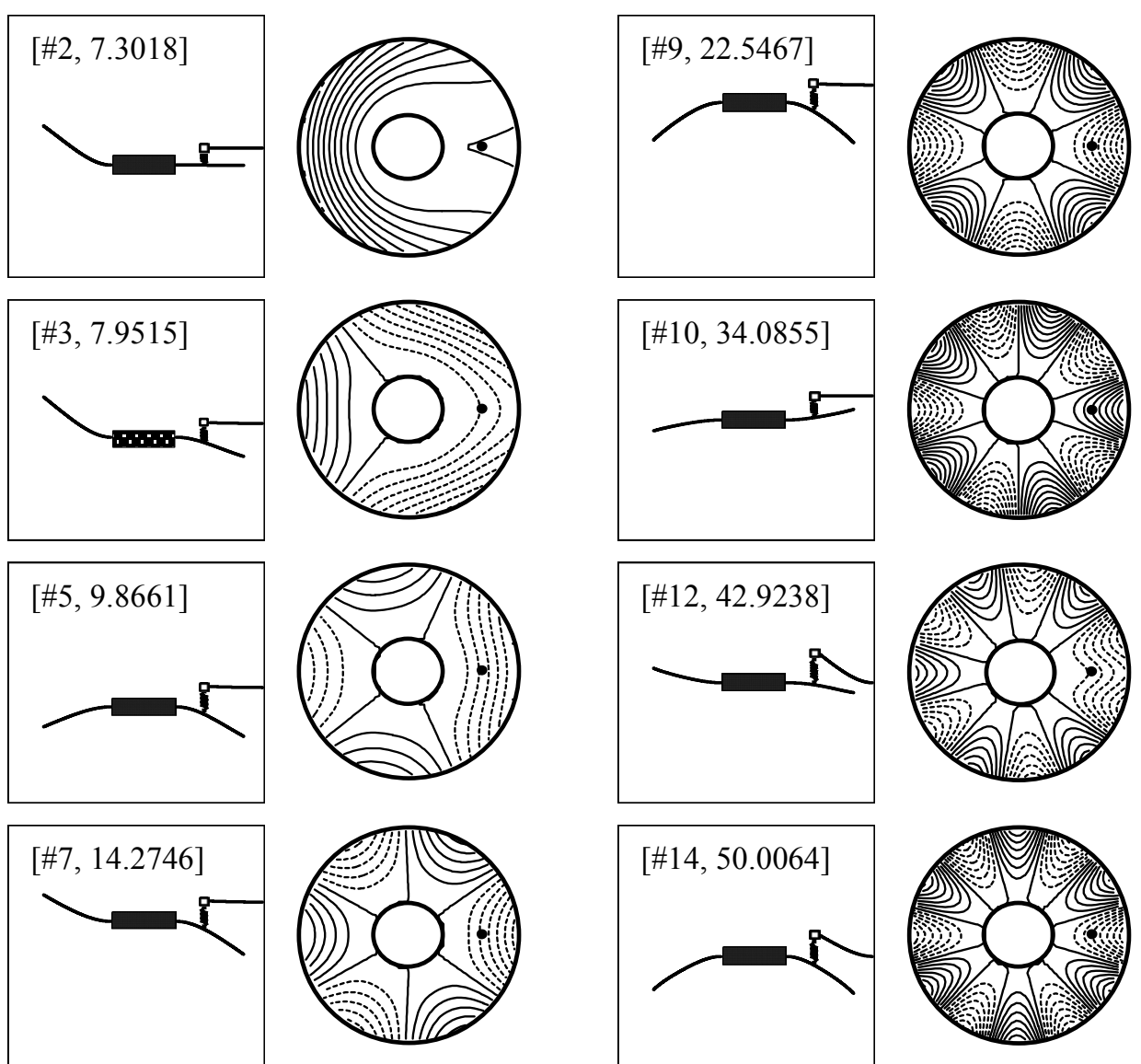

Fig. 5: The induced modes of a disk-head system.

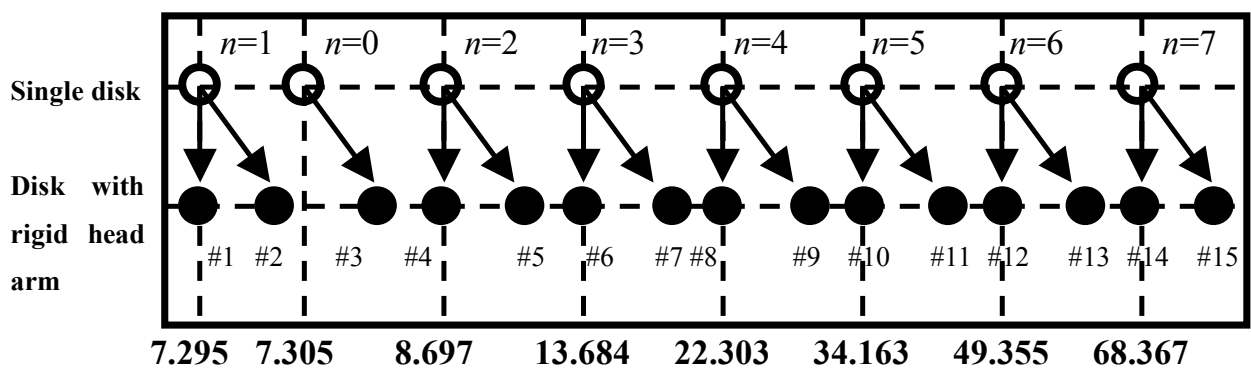
Natural frequencies $\omega^{*}$
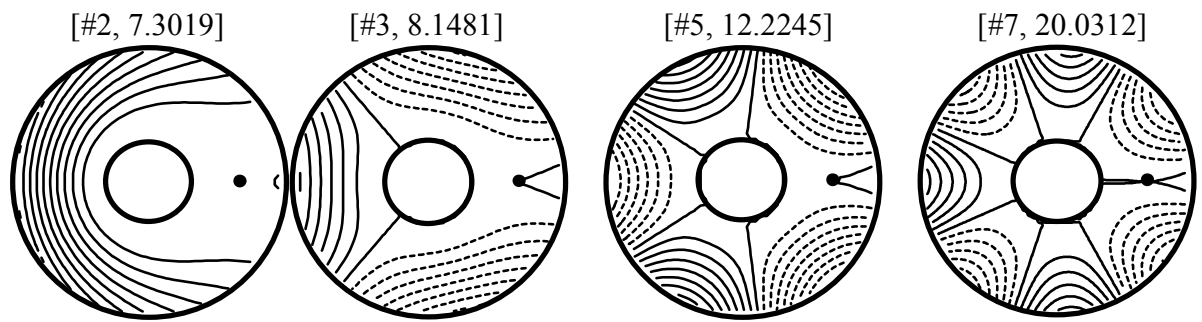

[\#9, 31.0621]

[\#11, 45.2416]

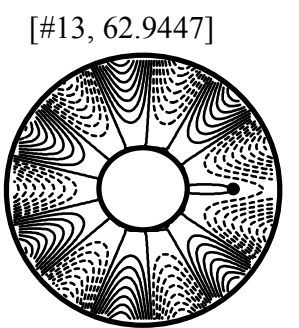

[\#15, 84.9215]

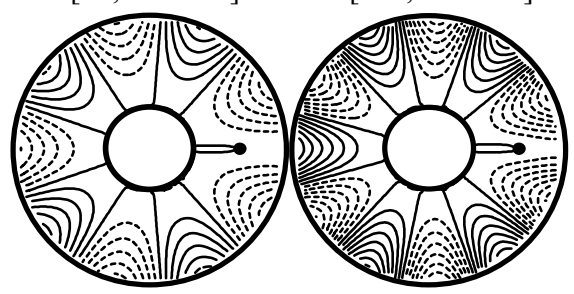

Fig. 6: The induced modes with rigid head arm.

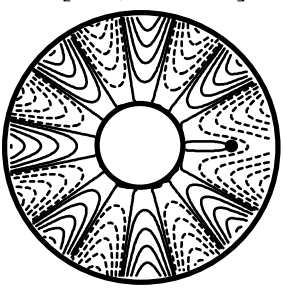


Figure 7(a) illustrates the disk's frequencies varying with the air spring constant $k^{*}$ for $\Omega^{*}=0$ and $r_{0}=(a+b) / 2$. As seen, when $k^{*}$ increases the induced modes' frequencies increase as well. From Fig. 7(a) it seems that $k^{*}$ changes the natural frequencies insignificantly. It is true but if one explores the change of mode shapes, e.g., $n=0$, and $n=1$ modes for $k^{*}=0.1 \sim 5.0$ as shown in Fig. 7(b), drastically change of mode shapes are observed. For as least as a $k^{*}=0.5$, the $n=0$ mode disappears and it is replaced by a new mode close to $n=1$.
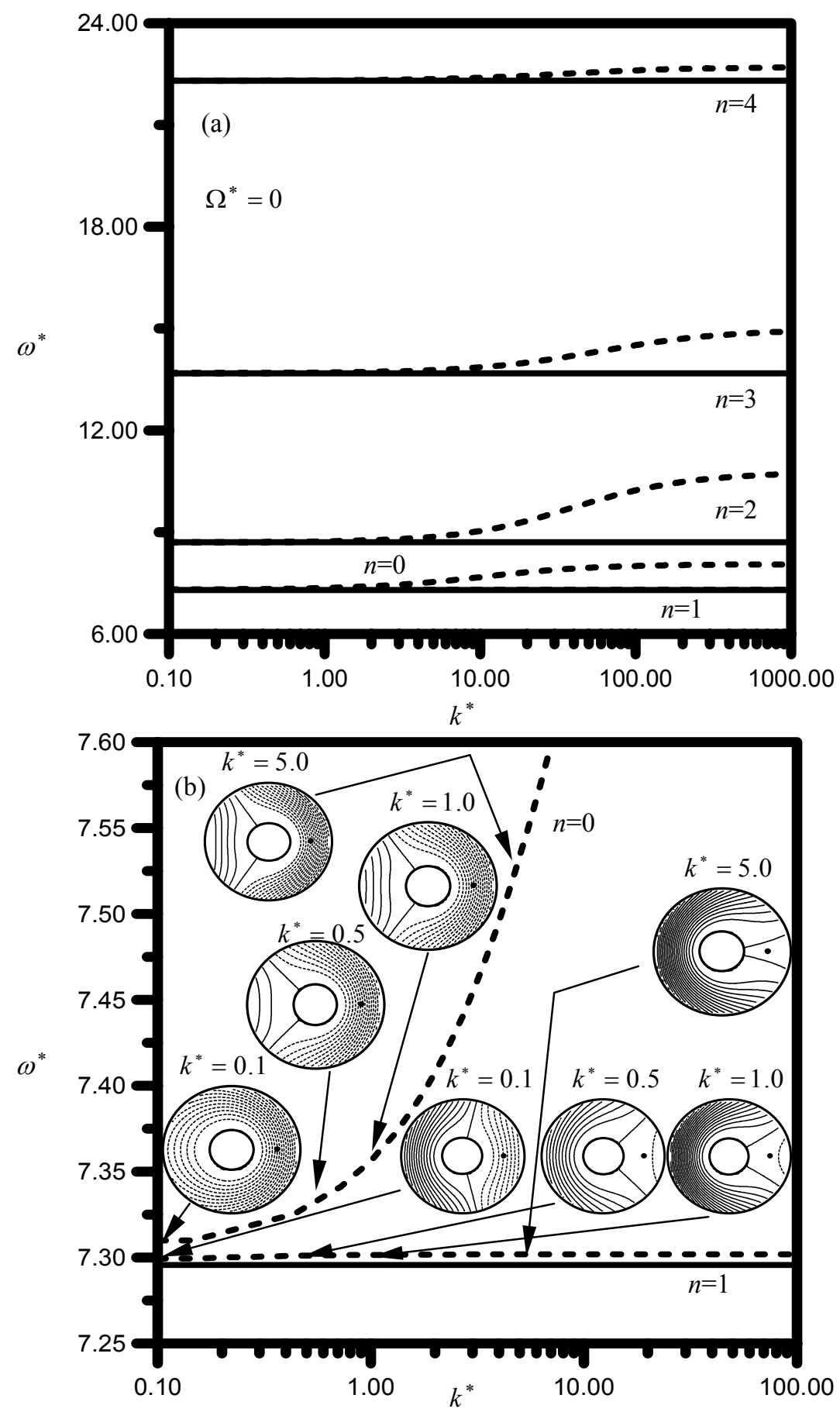

Fig. 7: Variations of natural frequencies with air spring constant.

To realize the influence of head flexibility on the coupling between disk and head, the authors here define a rigidity ratio of head arm to disk $R=\left(E_{b} I_{b}\right) /\left(D L_{b}\right)$. Figure 8 shows 
how the natural frequencies vary with $R$. As seen, the induced modes increase with $R$ as expected. The influence of arm rigidity to the natural frequencies is relatively weak because the air spring is relatively flexible than both of the disk and head arm. Figure 9 shows the effects of the head location for a $\Omega^{*}=0, k^{*}=50$ case. As expected, the natural frequencies change more significantly at the outer than the inner.

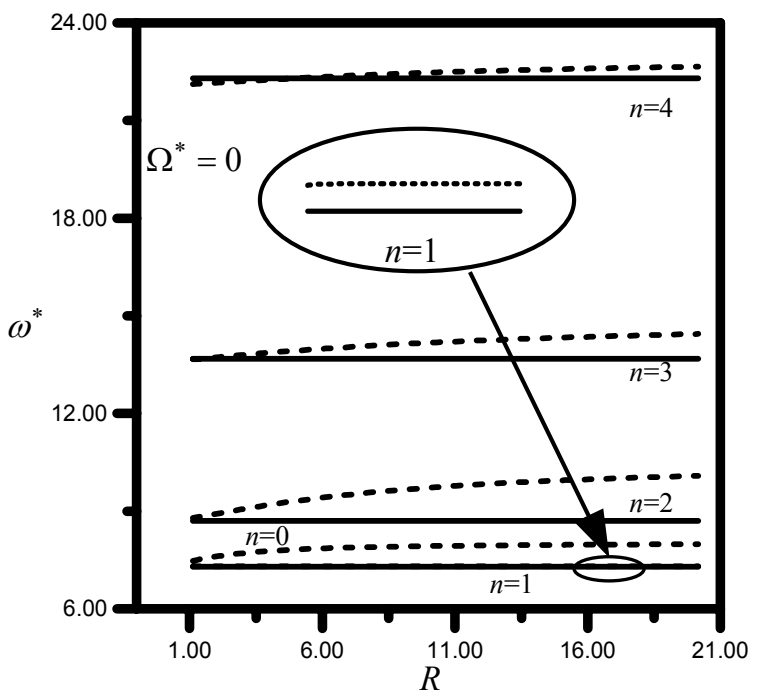

Fig. 8: Variations of natural frequencies with rigidity ratio.

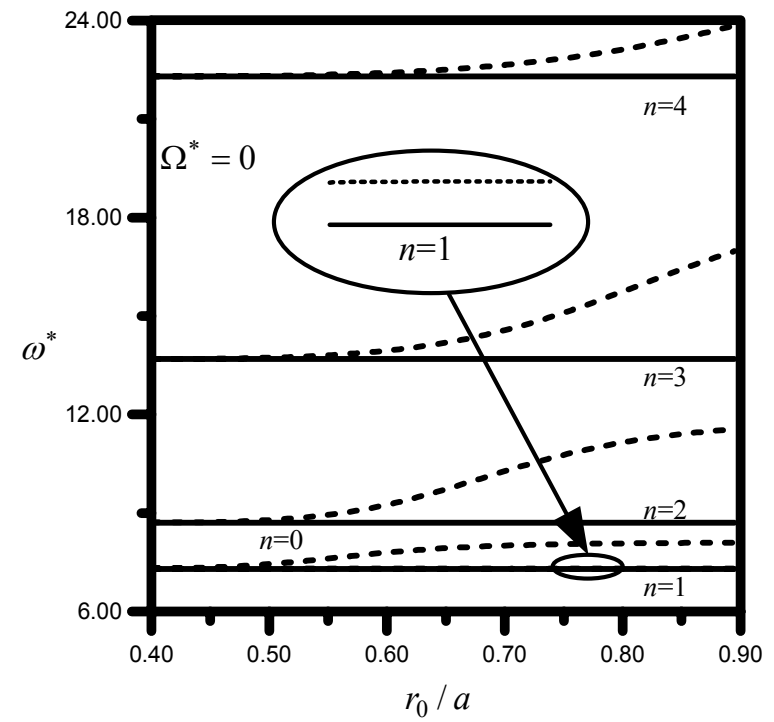

Fig. 9: Variations of natural frequencies with head location.

Finally, Fig. 10 shows the disk's frequency loci due to spinning. Three different spring constants are set to respectively denote the weak, the moderate, and the strong coupling. For a weak coupling $\left(k^{*}=1\right)$, the disk-head's natural frequencies almost coincide with the single disk's natural frequencies (dotted curves). At a moderate coupling $\left(k^{*}=50\right)$, there exist more starting points at $\Omega^{*}=0$ loci and it is referred to as frequency bifurcation phenomenon caused by head assembly. The loci show curve veering and crossing phenomena. This is a peculiar feature often seen in a rotating structure ${ }^{(26,28)}$. For strong coupling $\left(k^{*}=1000\right)$, the phenomena remain but the loci curves are farther separated from the beginning and repel one another around crossings. 

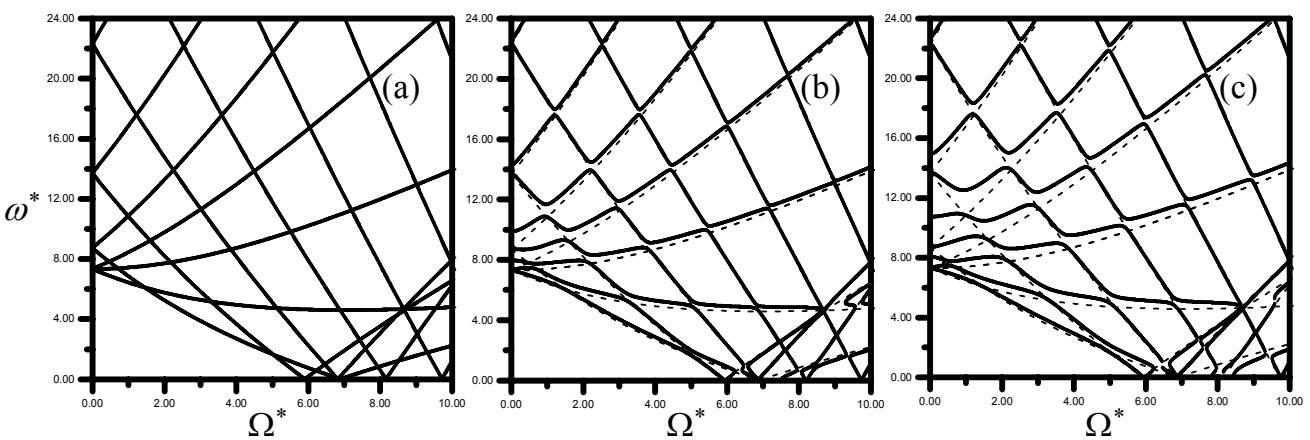

Fig. 10: Frequency loci with respect to spinning speed for (a) weak coupling,

(b) moderate coupling, and (c) strong coupling.

\section{Concluding Remarks}

A spinning disk-head assembly system representing a computer storage device was investigated via a new approach. The equations of motion of a spinning disk and of a head assembly were first derived and the receptance method followed to join disk and head assembly. The natural frequencies and mode shapes of disk-head system were then solved for. From the numerical results, the authors have arrived at the following conclusions. (i) The head assembly induces more modes for $n>0$ and causes bifurcations of frequencies. (ii) Increasing the head rigidity, by stiffening arm or removing air spring, the system's natural frequencies increase and it tends to yield the head attachment as a node. (iii) The air spring affects slightly the natural frequencies but changes the mode shapes significantly, particularly on lower $n$ modes. In words, the system's natural frequencies may not be altered by the heads' weak coupling but the modes are very different. (iv) Disk spinning is found not only to change the system's natural frequencies but also to pull the frequency loci passing through the crossings of disk's natural frequencies.

\section{References}

(1) Lamb, H., and Southwell, R.V., The Vibrations of a Spinning Disk, Proceedings of the Royal Society, Vol. 99 (1921), pp. 272-280.

(2) Mote, C.D.Jr., Free Vibrations of Initially Stressed Circular Disks, ASME J. Engi. for Industry, Vol. 87 (1965), pp. 258-264.

(3) Adams, G.G., Critical Speeds for a Flexible Spinning Disk, Int. J. Mech. Sci., Vol. 29 (1987), pp. 525-531.

(4) Eversman, W., and Cloyd, G.B., The Coupled Transverse Vibrations of a Spinning Membrane Disk with a Central Hub, J. Sound Vibr., Vol. 24, No. 1 (1972), pp. 1-15.

(5) Phylactopoulos, A., and Adams, G.G., Transverse Vibration of a rectangularly Orthotropic Spinning Disk, Part 1: Formulation and Free Vibration, ASME J. Vibr. Acoust., Vol. 121 (1999), pp. 273-279.

(6) Phylactopoulos, A., and Adams, G.G., Transverse Vibration of a rectangularly Orthotropic Spinning Disk, Part 2: Forced Vibration and Critical Speeds, ASME J. Vibr. Acoust., Vol. 121 (1999), pp. 280-285.

(7) Chung, J., Heo, J.W., and Han, C.S., Natural Frequencies of a Flexible Spinning Disk Misaligned with the Axis of Rotation, J. Sound Vibr., Vol. 260 (2003), pp. 763-775.

(8) Lee, H.P., and Ng, T.Y., Vibration and Critical Speeds of a Spinning Annular Disk of Varying Thickness, J. Sound Vibr., Vol. 187, No. 1 (1995), pp. 39-50.

(9) Eid, H., and Adams, G.G., Critical Speeds and the Response of a spinning Disk to a Stationary Load Using Mindlin Plate Theory, J. Sound Vibr., Vol. 290 (2006), pp. 209-222.

(10) Sinha, S.K., Free Vibrations of a Thick Spinning Annular Disk with Distributed Masses at the Outer Edge, J. Sound Vibr., Vol. 122 (1988), pp. 217-231. 
(11) Sinha, S.K., On Free Vibrations on a Thin Spinning Disk Stiffened with an Outer Reinforcing Ring, ASME J. Vibr. Acoust. Stress Reli. in Design, Vol. 110 (1988), pp. 507-514.

(12) DasGupta, A., and Hagedorn, P., Critical Speeds of a Spinning Thin Disk with an External Ring, J. Sound Vibr., Vol. 283 (2005), pp. 765-779.

(13) Yang, S.M., Vibration of a Spinning Annular Disk with Coupled Rigid-Body Motion, J. Vibr. and Acoust., Vol. 115 (1993), pp. 159-164.

(14) Young, T.H., and Lin, C.Y., Stability of a Spinning Disk Under a Stationary Oscillating Unit, J. Sound Vibr., Vol. 298 (2006), pp. 307-318.

(15) Chivens, D.R., and Nelson, H.D., The Natural Frequencies and Critical Speeds of a Rotating, Flexible Shaft-Disk System, ASME J. Engi. for Industry, Vol. 97 (1975), pp. 881-886.

(16) Shahab, A.A.S., and Thomas, J., Coupling Effects of Disc Flexible on the Dynamic Behavior of Multi Disc-Shaft System, J. Sound Vibr., Vol. 114 (1987), pp. 435-452.

(17) Yang, C.H., and Huang, S.C., The Influence of Disk's Flexibility on Coupling Vibration of Shaft-Disk-Blades System, J. Sound Vibr., Vol. 301 (2007), pp. 1-17.

(18) Benson, R.C., and Bogy, D.B., Deflection of a Very Flexible Spinning Disk Due to a Stationary Transverse Load, ASME J. Applied Mech., Vol. 45 (1978), pp. 636-642.

(19) Stahl, K.J., and Iwan, W.D., On the Response of a Two-Degree-Freedom Rigid Disk with a Moving Massive Load, ASME J. Applied Mech., Vol. 40 (1973), pp. 114-120.

(20) Stahl, K.J., and Iwan, W.D., On the Response of an Elastically Supported Rigid Disk with a Moving Massive Load, Int. J. Mech. Sci., Vol. 15 (1973), pp. 535-546.

(21) Iwan, W.D., and Moeller, T.L., The Stability of a Spinning Elastic Disk with a Transverse Load System, ASME J. Applied Mech., Vol. 43 (1976), pp. 485-490.

(22) Shen, I.Y., andMote, C.D. Jr., On the Mechanisms of Instability of a Circular Plate Under a Rotating Spring-Mass-Dashpot System, J. Sound Vibr., Vol. 148 (1991), pp. 307-318.

(23) Chen, J.S., and Bogy, D.B., Effects of Load Parameters on the Natural Frequencies and Stability of a Flexible Spinning Disk with a Stationary Load System, ASME J. Applied Mech., Vol. 59 (1992), pp. S230-S235.

(24) Chen, J.S., and Bogy, D.B., Natural Frequencies and Stability of a Flexible Spinning Disk-Stationary Load System with Rigid-Body Tilting, ASME J. Applied Mech., Vol. 60 (1993), pp. 470-477.

(25) Liang, D.S., Wang, H.J., and Chen, L.W., Vibration and Stability of Rotating Polar Orthotropic Annular Disks Subjected to a Stationary Concentrated Transverse Load, J. Sound Vibr., Vol. 250 (2002), pp. 795-811.

(26) Huang, S.C., and Chiou, W.J., Modeling and Vibration Analysis of Spinning-Disk and Moving-Head Assembly in Computer Storage System, ASME J. Vibr. Acoust., Vol. 119 (1997), pp. 185-191.

(27) Yu, S.C., and Huang, S.C., Vibration Attenuation via Constrained Layer Damping and Dashpot on a Spring-Mass-Plate System, J. Sound Vibr., Vol. 259, No. 5 (2003), pp. 1181-1198.

(28) Huang, S.C., and Hsu, B.S., Theory of Receptance Applied to Modal Analysis of a Spinning Disk with Interior Multi-Point Supports, ASME J. Vibr. Acoust., Vol. 114 (1992), pp. 468-476. 